\title{
Cranioplasty using Titanium Mesh Versus Acrylic Bone Cement: Short-term Outcomes and Complications
}

\author{
Mohab Darwish, Waleed Zidan Nanous
}

\begin{abstract}
Department of Neurosurgery, Minia University Hospital, Minia, Egypt.
\end{abstract}

Correspondence to: Mohab Darwish, Department of Neurosurgery, Minia University Hospital, Minia, Egypt.

Email:

mohab.darwish@mu.edu.eg

Received: 25 December 2020

Accepted: 4 January 2021

\section{Abstract:}

Background: Cranioplasty is a reconstructive procedure to restore bone anatomy and repair skull defects. Optimum reconstruction could be a challenge for neurosurgeons, and therefore the strategy to attain the ideal result remains a subject of discussion. Aim: we aimed at comparing two completely different prostheses in reconstructing calvarial bone defects, titanium mesh and polymethyl methacrylate (PMMA) bone cement. We looked for the differences in the cosmetic and functional outcomes as well as the prosthesis-related complications. Patients and Methods: This was a randomized prospective study on the first forty successive adult patients with calvarial skull defects of different etiologies, sites and sizes admitted and operated upon at neurosurgery department, Minia University hospital between January 2017 and December 2018. We divided patients into 2 groups, Group1: 20 patients were operated upon using Titanium mesh and Group 2: 20 patients were operated upon using (PMMA) acrylic bone cement implants. Results: Regarding cosmetic appearance, functional outcome, and improvement of the clinical symptoms (syndrome of trephined), Cranioplasty using titanium mesh and acrylic bone cement proved to have nonsignificant differences in the reconstruction of calvarial skull defects of different etiologies. However, there is a statistically significant difference between both materials regarding complications especially with large skull defects $\left(\geq 25 \mathrm{~cm}^{2}\right)$. Conclusion: there is no statistical difference between both materials regarding cosmetic and functional outcomes. However, large bone defects $\left(\geq 25 \mathrm{~cm}^{2}\right)$ are better treated with titanium mesh due to lower incidence of complications.

Key words: cranioplasty, titanium mesh, bone cement 



\section{List of Abbreviations:}

Decompressive craniectomy (DC)

Sinking skin flap syndrome (SSFS)

Polymethyl methacrylate (PMMA)

\section{Introduction}

Cranioplasty could be a reconstructive procedure to restore bone anatomy and repair skull defects. Optimum reconstruction could be a challenge for neurosurgeons, and therefore the strategy to attain the ideal result remains a subject of discussion. The most common causes of bone defects include depressed skull fractures, decompressive craniectomy (DC), invading tumors, congenital and inflammatory lesions. ${ }^{(1)}$

Many characteristics are urged to explain the best alloplastic material for cranioplasty including biocompatibility, tissue tolerance, simplicity of manufacture, simple sterilization, low thermal conduction, radiolucency, lightweight, resistance to infections, low price and being easy to use. ${ }^{(2,3,4)}$

Sinking skin flap syndrome (SSFS) is defined as serious disabling neurologic deficits and impairment of general status with concave deformity and relaxation of the skin flap and it tends to develop several weeks to months after large craniectomy. ${ }^{(5)}$ Symptoms of SSFS include headache, vertigo, tinnitus, fatigue, loss of concentration, loss of memory, depression, and convulsions. Cranioplasty can improve neurological status in patients with SFSS. ${ }^{(4)}$

Titanium is bio acceptable with no inflammatory reaction. It also showed good resistance to infection, even when in contact with the Paranasal sinuses. ${ }^{(5)}$ Bone cement has gained increasing attraction for calvarial reconstruction over the past few years, given their ease of application and ability to conform to most defect shapes. ${ }^{(6)}$

We aimed at comparing two completely different prostheses in the reconstruction of the calvarial bone defects, titanium mesh and polymethyl methacrylate (PMMA) bone cement. We looked for differences in the cosmetic and functional outcomes as well as the prosthesis-related complications.

\section{Patients and Methods:}

This was a randomized prospective comparative study on the first successive forty adult patients with calvarial skull defects of different etiologies, sites and sizes. After approval of the local ethical committee, patients were admitted and operated upon at neurosurgery department, Minia university 
hospital. The study was between January 2017 and December 2018.

We divided the forty patients into two groups. Group one, comprises twenty patients operated with titanium mesh (Fig. 1-2). Group two, involves patients operated with intraoperatively molded acrylic bone cement (Fig. 3).

We had 28 male patients and 12 female patients in both groups with ages ranged between 14 and 55 years old with mean/SD (28.6 $\pm 12.1 \mathrm{yrs})$. Regarding etiology of the bone defect, 27 patients were due to compound depressed fractures, 10 patients were due to neoplastic lesions, and 3 patients were post decompressive craniectomy. Patients with compound depressed fracture were operated upon at least 3 months after elevation of the depressed bones and dural repair to minimize the risk of infection. Patients with CSF leak were treated first for this leak before bone grafting by at least 3 months. All patients had clean recipient bone site with no clinical or radiological pictures of infection. All patients had postoperative CT scan with $3 \mathrm{D}$ reconstruction. All patients were followed up for at least 6-12 months.

Patients in both groups were compared regarding their cosmetic and functional results, sizes of the bone defects and procedure-related outcomes and complications. Cosmetic and functional outcomes were assessed according to Honeybul et al. (7) as follows: complete success, partial success, satisfactory, partial failure, and complete failure.

\section{Results}

\section{Cosmetic outcome:}

According to doctor's assessment, group one has 18 patients with complete success and 2 patients with partial success while Group two has 17 patients with complete success and 3 patients with partial success.

According to patient's assessment, group one has 18 patients with complete success, 1 patient with partial success and 1 patient with satisfactory result. Group two, comprises 15 patients with complete success, 4 patients with partial success and 1 patient with satisfactory result.

There is no statistically significant difference (p-value >0.05) between both groups regarding cosmetic assessment by doctor and patients indicating that both procedures had the same cosmetic outcome.

\section{Functional outcome (Restoration of} cranial coverage on clinical palpation):

According to doctor's assessment, group one has 17 patients with complete success and 3 
patients with partial success. Group two has 16 patients with complete success, 2 patients with partial success and 2 patients with satisfactory result.

According to patient's assessment, group one has 16 patients with complete success and 4 patients with partial success. Group two has 15 patients with complete success, 2 patients with partial success and 3 patients with satisfactory result.

Statistically, there is no significant difference (p-value >0.05) between the two groups regarding functional assessment by doctor and patients indicating that both procedures had the same functional outcome.

\section{3) Early complications $(<3 \quad$ weeks}

\section{postoperative):}

In group one, two patients had subgaleal collection while in group two, 8 patients had subgaleal collection and 2 patients had early postoperative superficial infection.

There is a statistically significant difference ( $\mathrm{p}$-value $=0.017$ ) between both groups regarding early complications.

\section{4) Late complications ( $>3$ weeks): (Fig. 4)}

We did not report any late complications in patients operated with titanium mesh. In patients operated with bone cement, three patients had late wound infection that responded very well to a ten-day broad- spectrum antibiotic course. Two patients had late wound infection that did not respond to the antibiotic course and required bone graft removal. One patient had his bone graft exposed and removed.

Statistically, there is a significant difference ( $\mathrm{p}$-value $=0.008$ ) between the two groups regarding late complication.

\section{Association between skull defect size and postoperative complications:}

In group one, 2 out of 5 patients with defect size $\geq 25 \mathrm{~cm}^{2}$ had "early" post-operative complications in the form of subgaleal collection. None of this group's patients had any late complication regardless the size of the defect (6 patients with defect size $\leq 9 \mathrm{~cm}^{2}, 9$ patients with defect size $10-24 \mathrm{~cm}^{2}$ and 5 patients with defect size $\geq 25 \mathrm{~cm}^{2}$ ).

Group two, with defect size $\leq 9 \mathrm{~cm}^{2}, 3$ out of 10 patients had early postoperative subgaleal collection and two other patients had superficial wound infection treated conservatively but no late complications in all of them. With defect size $10-24 \mathrm{~cm}^{2}, 2$ out of 5 patients had early postoperative sub glial collection, and late infection treated medically with no need for graft removal. With defect size $\geq 25 \mathrm{~cm}^{2}, 3$ out of 5 patients had early postoperative subgaleal collection. One of 
them had late infection treated medically with no need for graft removal. The other two had late deep infection that required graft removal. Only one had graft exposure and removal.

Statistically, there is a significant correlation ( $p$-value $=0.007)$ between skull defect size and

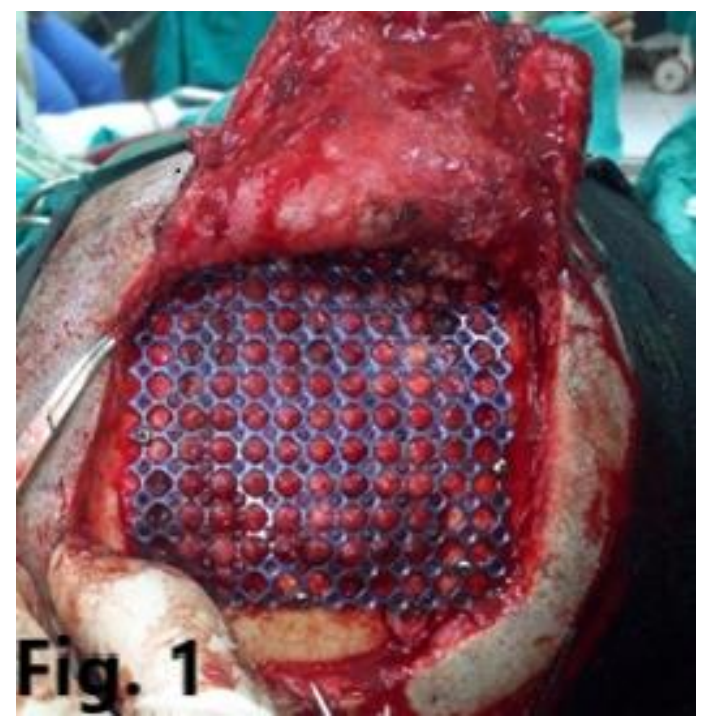

Fig. 1 shows bone defect after compound depressed fracture covered with titanium mesh with postoperative $3 \mathrm{D} \mathrm{CT}$ postoperative complications with higher percentage of complications among patients with defect size $\geq 25 \mathrm{~cm}^{2}$ especially when this defect is covered with bone cement.

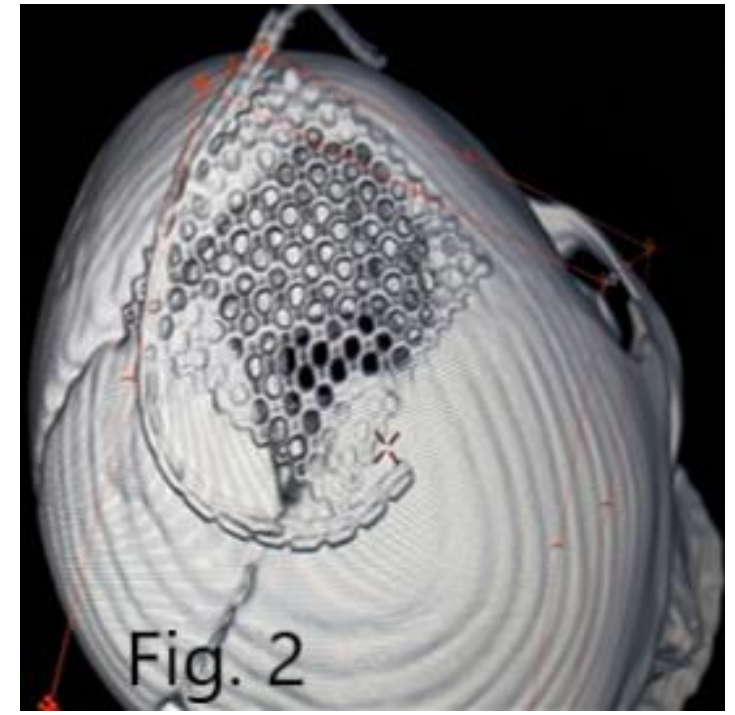

Fig. 2: shows well-fit implant

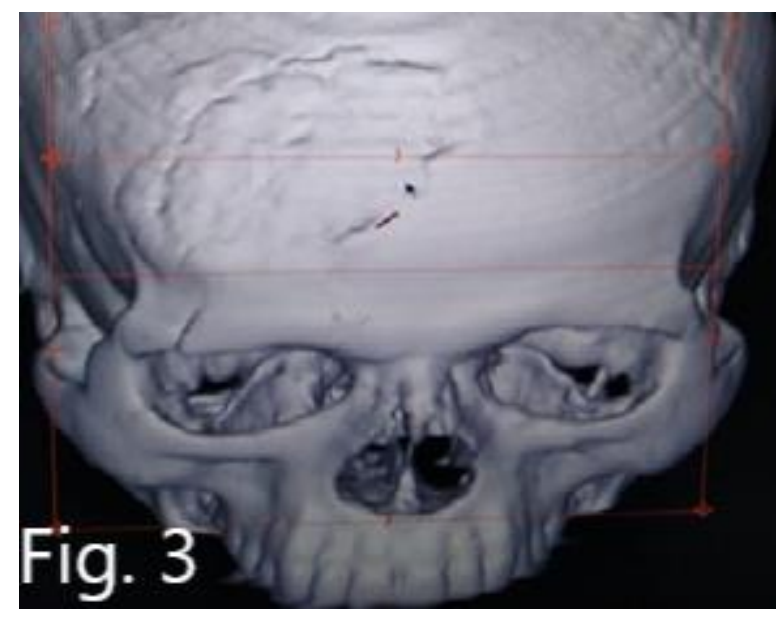

Fig. 3 compound depressed fracture covered with bone cement with postoperative 3D CT shows well-fit implant 


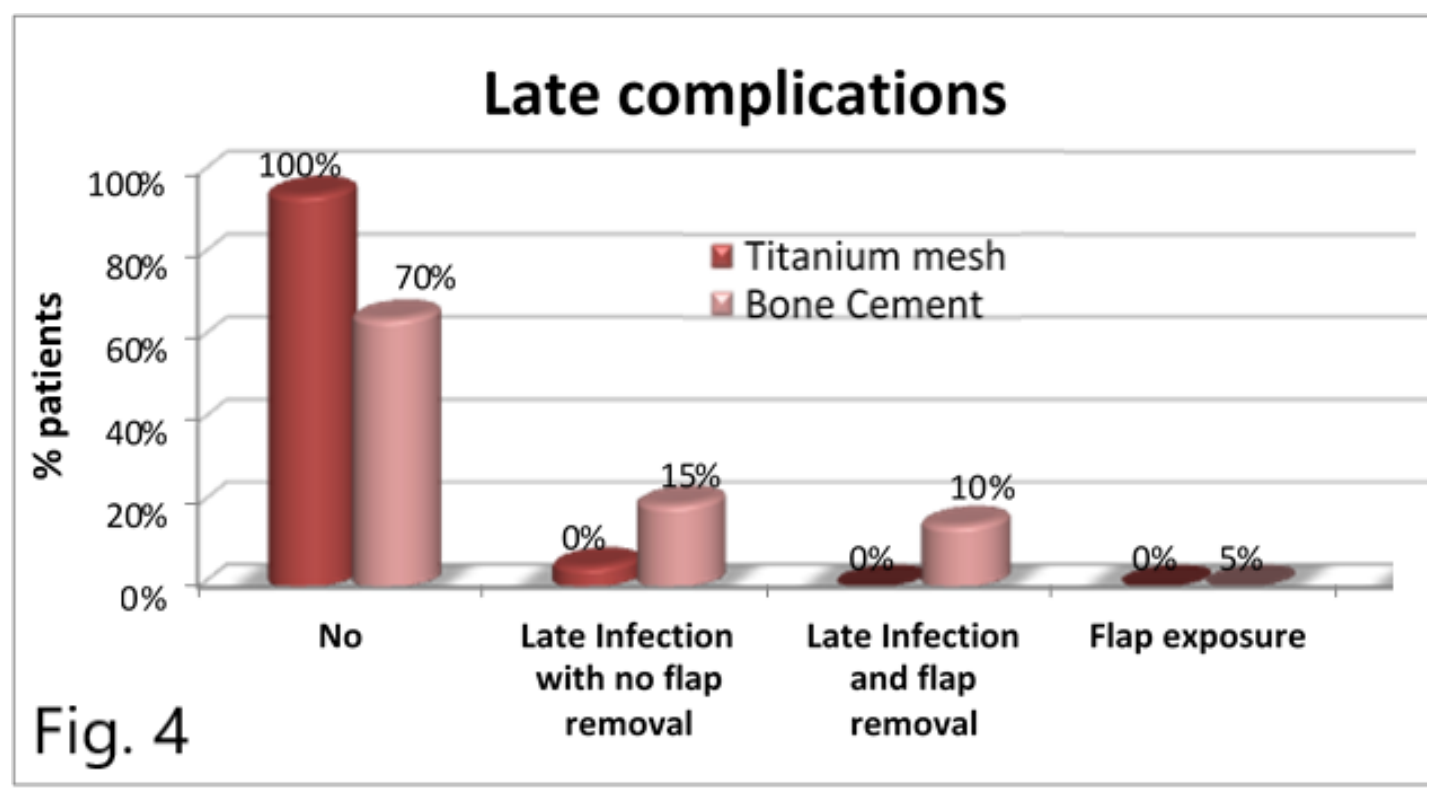

Figure 4: Comparison of late complications in both study groups

\section{Discussion}

In our study, we assessed the postoperative cosmetic appearance in all patients from their and our perspectives. Both titanium mesh and bone cement gave approximately the same cosmetic and functional results with no statistical differences. These results are consistent with multiple studies by some other authors. ${ }^{(7,8,9,10,11)}$

However, regarding complications, both groups were statistically different regarding both the type of used material and the size of defect to be covered. Regarding early complications (within $1^{\text {st }} 3$ weeks), only two patients treated with titanium mesh had subgaleal collection compared to eight patients treated with bone cement. Moreover, two patients treated with bone cement had early infection that resolved with medical treatment compared to none in the titanium mesh group. Regarding late complications (> 3 weeks), none of the patients treated with titanium mesh had late complications compared to six patients in the bone cement group with three out of them required graft removal.

Regarding titanium mesh, our results are similar to Honeybul et al., $2017^{(7)}$ who had only one patient (out of 31) with late infection who needed titanium mesh re-implantation after 
antibiotic course. Our results also agree with another study ${ }^{(8)}$ which analyzed titanium mesh on 56 patients and found that an abscess developed in one patient $(1.7 \%)$ who received high-dose steroids for 72 hours before reconstruction. The case was treated with broadspectrum intravenous antibiotics, bedside incision, and drainage and did not require removal of the titanium mesh. However, these results disagree with another study done somewhat later ${ }^{(9)}$, where it was found that in 151 patients underwent cranioplasty using titanium mesh, 10 patients had "early complications" and 29 patients had "late complications" in the form of seromas and infections. We attribute this disagreement to the difference in the number of treated patients (20 vs 151) and the large average defect surface area in their study $\left(67.5 \mathrm{~cm}^{2}\right)$.

Regarding bone cement, our results are similar to the study done on $2003^{(10)}$ which proved that in 312 patients underwent 449 cranioplasty procedures; the use of bone cement was associated with the highest rate of complications especially for large bone defects. It seems to induce an immune guided delayed inflammatory reaction that leads to thinning of the skin and exposure of the material, making secondary repair difficult. Our results are not similar to the study showing excellent cosmetic reconstruction with PMMA with no prosthesisrelated complications ${ }^{(11)}$. However, his study was conducted on patients with only small and medium sized defects $(<8 \mathrm{~cm})$. In the study done on $2004^{(12)}$, showed that there were 5 out of 48 patients (10\%) post-operative complications with bone cement in the form infection and subgaleal collection. However, most of their patients were children with small congenital defects unlike our patients who were only adult and mostly post-traumatic. Also, in another study ${ }^{(13)}, 10$ out of 61 patients (15\%) had post-operative complications with bone cement. These last two studies used the hydroxyapatite bone cement.

In a study one on $2016^{(14)}$ it was proved that over a 5-year period, 672 patients underwent Retro mastoid craniectomy (RMC) reconstructed with cement or titanium mesh. It was found that with using titanium mesh there were 38 wound complications, including 18 (5.4\%) patients with infection and $20(6 \%)$ patients with CSF leak ${ }^{(14)}$. With bone cement, two patients $(0.6 \%)$ experienced wound infection and no patients (0\%) had CSF leak. This disagreement could be explained by the large number of patients in his study and by the fact that all of his patients underwent RMC for surgical treatment of cranial nerve pathology, including microvascular decompression for cranial nerve neuralgias, and for the resection of tumors involving the cranial nerves and lateral brainstem. In addition, calcium phosphate bone cement was used ${ }^{(14)}$.

In addition to the material-related complications, our results showed statistically significant size-related complications. This was 
more evident in large sized defects $\left(>25 \mathrm{~cm}^{2}\right)$ especially when treated with PMMA bone cement. Same results found ${ }^{(3)}$, where bone cement was analyzed on 16 patients who underwent correction of large full-thickness $\left(\geq 25 \mathrm{~cm}^{2}\right)$ skull defects found major complications occurred in eight of 16 patients, with one occurring as late as 6 years postoperatively. Nearly, similar results found by other researchers ${ }^{(10)}$ where it was concluded that covering large defects with bone cement should be approached with caution.

\section{Conclusion}

Regarding cosmetic appearance, functional outcome, and improvement of the clinical symptoms (syndrome of trephined), cranioplasty using Titanium mesh and PMMA bone cement proved to have non-significant difference in the reconstruction of calvarial skull defects. However, titanium mesh provides fewer rates of complications than bone cement especially with large skull defects $\left(\geq 25 \mathrm{~cm}^{2}\right)$.

Being cheaper and more malleable, bone cement is favored in small-sized bone defects. On the other hand, the lower incidence of complications with large defects using titanium mesh gives it higher priority on choosing the proper procedure preoperatively.

This study is preliminary. Both materials are compared for short period of time $(\leq 12$ months). This study will be integrated into another long-term comparative study of these two materials with involvement of pediatric population.

\section{References}

1. De Bonis $\mathrm{P}$, Frassanito $\mathrm{P}$, Mangiola A, Nucci CG, Anile C, Pompucci A. Cranial repair: how complicated is filling a "hole"? J Neurotrauma. 2012;29(6):10716.

2. Zanotti B, Zingaretti N, Verlicchi A, Robiony M, Alfieri A, Parodi PC. Cranioplasty: Review of Materials. J Craniofac Surg. 2016 Nov;27(8):20612072.

3. Zins JE, Moreira-Gonzalez A, Papay FA. Use of calcium-based bone cements in the repair of large, full-thickness cranial defects: a caution. Plast Reconstr Surg. 2007 Oct;120(5):1332-1342.

4. Akins PT, Guppy KH. Sinking skin flaps, paradoxical herniation, and external brain tamponade: a review of decompressive craniectomy management. Neurocrit Care. 2008;9(2):269-76.

5. Aatman MS, Henry J, Stephen S. Materials used in cranioplasty: a history and analysis. Neurosurg Focus. 2014 Apr;36(4):E19.

6. Piazza M, Grady MS. Cranioplasty. Neurosurg Clin N Am. 2017 Apr;28(2):257-265.

7. Honeybul S, Morrison DA, Ho KM, Lind CR, Geelhoed E. A randomized 
controlled trial comparing autologous cranioplasty with custom-made titanium cranioplasty. J Neurosurg. 2017 Jan;126(1):81-90.

8. Gear AJL, Lokeh A, Aldridge JH, Migliori MR, Benjamin CI, Schubert W. Safety of Titanium Mesh for Orbital Reconstruction. Ann Plast Surg. 2002 Jan;48(1):1-7; discussion 7-9.

9. Williams L, Fan K, Bentley R. Custommade titanium cranioplasty: early and late complications of 151 cranioplasties and review of the literature. Int $\mathrm{J}$ Oral Maxillofac Surg. 2015 May;44(5):599608.

10. Moreira-Gonzalez A, Jackson IT, Miyawaki T, Barakat K, DiNick V. Clinical outcome in cranioplasty: critical review in long-term follow-up. $\mathrm{J}$ Craniofac Surg. 2003 Mar;14(2):144-53.

11. Abd El-Ghani, W.M.A. Cranioplasty with polymethyl methacrylate implant: solutions of pitfalls. Egypt J

Neurosurg 33, 7 (2018).

12. Magee WP Jr, Ajkay N, Freda N, Rosenblum RS. Use of fast-setting hydroxyapatite cement for secondary craniofacial contouring. Plast Reconstr Surg. 2004 Aug;114(2):289-97.

13. Burstein FD, Cohen SR, Hudgins R, Boydston W, Simms C. The use of hydroxyapatite cement in secondary craniofacial reconstruction. Plast Reconstr Surg. 1999 Oct;104(5):1270-5.

14. Foster KA, Shin SS, Prabhu B, Fredrickson A, Sekula Jr RF. Calcium phosphate cement cranioplasty decreases the rate of cerebrospinal fluid leak and wound infection compared with titanium mesh cranioplasty: retrospective study of 672 patients. World Neurosurg. 2016 Nov;95:414-418.

To cite this article: Mohab Darwish, Waleed Zidan Nanous. Cranioplasty using Titanium Mesh Versus Acrylic Bone Cement: Short-term Outcomes and Complications. BMFJ 2021; 38(1):146154. DOI: $10.21608 / \mathrm{bmfj} .2021 .54997 .1363$. 